TRANSACTIONS OF THE

AMERICAN MATHEMATICAL SOCIETY

Volume 350, Number 9, September 1998, Pages 3639-3655

S 0002-9947(98)01967-9

\title{
ON ZETA FUNCTIONS AND IWASAWA MODULES
}

\author{
JANGHEON OH
}

\begin{abstract}
We study the relation between zeta-functions and Iwasawa modules. We prove that the Iwasawa modules $X_{k\left(\zeta_{p}\right)}^{-}$for almost all $p$ determine the zeta function $\zeta_{k}$ when $k$ is a totally real field. Conversely, we prove that the $\lambda$-part of the Iwasawa module $X_{k}$ is determined by its zeta-function $\zeta_{k}$ up to pseudo-isomorphism for any number field $k$. Moreover, we prove that arithmetically equivalent CM fields have also the same $\mu^{-}$-invariant.
\end{abstract}

\section{INTRODUCTION}

Let $\zeta_{k}(s)$ be the zeta function attached to a number field $k$. When two number fields share a common zeta function, they are said to be arithmetically equivalent. Isomorphic fields have identical zeta functions. The first non-isomorphic arithmetically equivalent fields were discovered in 1925 by Gassmann [3]. If $k$ is isomorphic to any field $L$ with the same zeta function, that is, if $\zeta_{k}=\zeta_{L} \Rightarrow k \simeq L$, then $k$ is said to be arithmetically solitary. Robert Perlis [9] proved that any field $k$ of degree $[k: \mathbb{Q}] \leq 6$ is solitary. However, there are infinite families of $k, k^{\prime}$ of non-isomorphic arithmetically equivalent fields (see Perlis [9]).

In 1958, with the motivation from the theory of function fields, Iwasawa introduced his theory of $\mathbb{Z}_{p}$-extensions, and a few years later Kubota and Leopoldt invented $p$-adic $L$-functions. Iwasawa [5] interprets these $p$-adic $L$-functions in terms of $\mathbb{Z}_{p}$-extensions. In 1979, Mazur and Wiles proved the Main Conjecture, showing that $p$-adic $L$-functions are essentially the characteristic power series of certain Galois actions arising in the theory of $\mathbb{Z}_{p}$-extensions.

In Tate [12] and Turner [13], the following result is proved: let $k$ and $k^{\prime}$ be function fields in one variable over a finite constant field $F$ and $\zeta_{k}, \zeta_{k^{\prime}}$ be Dedekind zeta functions of $k, k^{\prime}$, respectively. Let $C, C^{\prime}$ be complete non-singular curves defined over $F$ with function fields isomorphic to $k, k^{\prime}$, and $J(C), J\left(C^{\prime}\right)$ the Jacobian varieties of $C, C^{\prime}$. Then the following are equivalent:

$$
\text { (1) } \zeta_{k}=\zeta_{k^{\prime}} \text {, }
$$

(2) $J(C)$ and $J\left(C^{\prime}\right)$ are $F$-isogenous.

Received by the editors April 16, 1996 and, in revised form, June 7, 1996 and October 23, 1996.

1991 Mathematics Subject Classification. Primary 11R23.

Key words and phrases. Iwasawa module, zeta function, $p$-adic $L$-function.

This paper is part of the author's Ph.D thesis. I would like to thank my adviser, W. Sinnott, for introducing me to this subject, for pointing out to me the key idea and for many valuable comments.

(C)1998 American Mathematical Society 
Komatsu [8] proved analogous results in the number field case. More explicitly, he proved the following result: Let $p$ be a rational prime number, $k$ and $k^{\prime}$ be

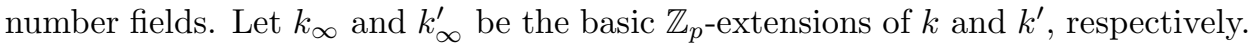
Let $X_{k}$ the Galois group of the maximal unramified abelian $p$-extension of $k_{\infty}$ over $k_{\infty}$. Then $\zeta_{k}=\zeta_{k^{\prime}}$ implies that $X_{k}$ and $X_{k^{\prime}}$ are isomorphic as $\Lambda$-modules for almost all prime numbers $p$. Adachi and Komatsu [1] proved a weaker converse statement of the above result: Let $k$ and $k^{\prime}$ be totally real number fields. Let $K_{\infty}$ be the cyclotomic $\mathbb{Z}_{p}$-extension of $k\left(\zeta_{p}\right), \Omega$ the maximal abelian $p$-extension of $K_{\infty}$ unramified outside $p$, and $Y_{k\left(\zeta_{p}\right)}$ the Galois group of $\Omega$ over $K_{\infty}$. If $Y_{k\left(\zeta_{p}\right)}$ is isomorphic to $Y_{k^{\prime}\left(\zeta_{p}\right)}$ for every prime $p$, then $\zeta_{k}=\zeta_{k^{\prime}}$.

In this paper, we will improve their results. First, we will prove that the Iwasawa modules $X_{k\left(\zeta_{p}\right)}$ for almost all primes $p$ determine the field $k$ up to arithmetic equivalence when $k$ is a totally real number field. In this case, the Main Conjecture relates the $p$-adic $L$-functions of $k$ and the Iwasawa module $X_{k}$. The $p$-adic $L$-functions give us enough information on the values of the zeta function of $k$ at negative integers. Combining this information and the functional equation, we can reconstruct the zeta function $\zeta_{k}$. The improvements in this paper of the result of Adachi and Komatsu are as follows: In this paper, we use a pseudo-isomorphism instead of an isomorphism, which seems to be natural in Iwasawa theory, and use the module $X_{k\left(\zeta_{p}\right)}^{-}$(see $\S 2$ for its definition), contained in the torsion part of $Y_{k\left(\zeta_{p}\right)}$, instead of $Y_{k\left(\zeta_{p}\right)}$. It is well-known that the rank of the free part of $Y_{k\left(\zeta_{p}\right)}$ determines the degree $[k: \mathbb{Q}]$ which we need in the proof of Theorem 1 of this paper. Here we prove that the smaller module $X_{k\left(\zeta_{p}\right)}^{-}$determines the degree $[k: \mathbb{Q}]$. The Main Conjecture is proved for odd primes, so the main point of Theorem 1 (see $\S 1$ ) is to prove the result of Adachi and Komatsu under the condition "for almost all prime $p$ " instead of "for every prime $p$ ".

Secondly, we will prove that the $\lambda$-parts of $X_{k}$ and $X_{k^{\prime}}$ are pseudo-isomorphic for any prime $p$ if number fields $k$ and $k^{\prime}$ are arithmetically equivalent. It is well-known that arithmetically equivalent number fields $k$ and $k^{\prime}$ have the same normal closure $L$ over $\mathbb{Q}$.

Let $G=\operatorname{Gal}(L / \mathbb{Q})$, and $L_{n}$ be the $n$-th layer of the basic $\mathbb{Z}_{p}$-extension $L_{\infty}$. Komatsu proved that $X_{k}$ is isomorphic to $X_{k^{\prime}}$ when $p$ does not divide $[L: \mathbb{Q}]$. The real obstruction in the case $p \mid[L: \mathbb{Q}]$ occurs when the basic $\mathbb{Z}_{p}$-extension $\mathbb{Q}_{\infty}$ of $\mathbb{Q}$ and $L$ are not linearly disjoint over $\mathbb{Q}$, since then the Galois group $G$ does not act on $X_{L, \lambda}$. To overcome the obstruction, we make $X_{L, \lambda}$ into a $\mathbb{Z}_{p}[G]$-module by tensoring so that we can show that $X_{k, \lambda}$ and $X_{k^{\prime}, \lambda}$ are pseudo-isomorphic as $\mathbb{Z}_{p}\left[\left[\operatorname{Gal}\left(L_{\infty} / L\right)\right]\right]$ modules. (Here the $\lambda$-part $X_{k, \lambda}$ is defined to be $X_{k} / \mathbb{Z}_{p}$-torsion $\left(X_{k}\right)$.) Further, we can show that $X_{k}$ is isomorphic to $X_{k^{\prime}}$ as an Iwasawa module when $p$ does not divide the order $[L: k]=\left[L: k^{\prime}\right]$. Moreover, we can strengthen our result when $k$ is a $\mathrm{CM}$ field. In fact, we prove that the characteristic polynomials of the modules $X_{k}^{-}$are the same for arithmetically equivalent CM fields $k$. This implies at least that their $\mu^{-}$-invariants are the same.

\section{Statement of the main theorems}

Let $k$ be a number field, and $S$ be a finite set of rational primes. Let $p$ be a prime not in $S$, let $\zeta_{p}$ be a $p$-th root of unity, denote $\operatorname{Gal}\left(k\left(\zeta_{p}\right) / k\right)$ by $\Delta$, and write $\mathbb{Z}_{p}\left[\left[\operatorname{Gal}\left(k\left(\mu_{p^{\infty}}\right) / k\right)\right]\right]$ by $\Lambda[\Delta]$, where $k\left(\mu_{p^{\infty}}\right)$ is the field obtained by adjoining all the $p$-power roots of unity to $k$. 
Theorem 1. Let $S$ be a finite set of primes. Let $k$ be a totally real number field. Suppose we know $X_{k\left(\zeta_{p}\right)}^{-}$as a $\Lambda[\Delta]$-module up to pseudo-isomorphism for all $p \notin S$; then we can determine the zeta function $\zeta_{k}$ of $k$.

Arithmetically equivalent fields $k$ and $k^{\prime}$ have the same normal closure $L$, and $k \cap \mathbb{Q}_{\infty}=k^{\prime} \cap \mathbb{Q}_{\infty}$, so that the Galois groups of the basic $\mathbb{Z}_{p^{-}}$-extensions $k_{\infty} / k$ and $k_{\infty}^{\prime} / k^{\prime}$ can be identified. Let

$$
\Lambda=\mathbb{Z}_{p}\left[\left[\operatorname{Gal}\left(k_{\infty} / k\right)\right]\right]=\mathbb{Z}_{p}\left[\left[\operatorname{Gal}\left(k_{\infty}^{\prime} / k^{\prime}\right)\right]\right]=\mathbb{Z}_{p}[[T]],
$$

and denote $\mathbb{Z}_{p}\left[\left[(1+T)^{p^{t}}-1\right]\right]$ by $\Lambda_{t}$. By the structure theorem of $\Lambda$-modules, every finitely generated torsion $\Lambda$-module $X$ is pseudo-isomorphic to a module of the form $\bigoplus_{i} \Lambda / p^{m_{i}} \bigoplus_{j} \Lambda / f_{j}^{n_{j}}(T)$, where $f_{j} \in \Lambda$ is a distinguished and irreducible polynomial prime to $p$. Define

$$
X_{\lambda}=X /\left(\mathbb{Z}_{p}-\text { torsion }(X)\right) .
$$

Note that $X_{\lambda}$ is pseudo-isomorphic to $\bigoplus_{j} \Lambda / f_{j}^{n_{j}}(T)$.

Theorem 2. Let $p$ be a prime number. Let $k$ and $k^{\prime}$ be number fields such that $\zeta_{k}=\zeta_{k^{\prime}}$. Then the Iwasawa modules $X_{k, \lambda}$ and $X_{k^{\prime}, \lambda}$ are pseudo-isomorphic as $\Lambda_{t}$-modules for some $t$. Moreover, $X_{k}$ is isomorphic to $X_{k^{\prime}}$ as a $\Lambda$-module if $p$ does not divide the degree $[L: k]=\left[L: k^{\prime}\right]$. If $k$ is a $C M$ field and $\zeta_{k}=\zeta_{k^{\prime}}$ for a number field $k^{\prime}$, then $k^{\prime}$ is also a CM field and char $X_{k}^{-}=$char $X_{k^{\prime}}^{-}$for any odd prime $p$.

\section{The Main Conjecture}

A $\mathbb{Z}_{p}$-extension of a number field $k$ is an extension $k_{\infty} / k$ with

$$
\operatorname{Gal}\left(k_{\infty} / k\right)=\Gamma \simeq \mathbb{Z}_{p}
$$

the additive group of $p$-adic integers. Let $\gamma$ be a topological generator of $\Gamma$. Let $A_{n}$ be the $p$-Sylow subgroup of the ideal class group of the unique $n$-th layer $k_{n}$ of the $\mathbb{Z}_{p}$-extension $k_{\infty} / k$. Then $X_{k}=\lim _{\leftarrow} A_{n}$ is isomorphic to the Galois group of the maximal unramified abelian $p$-extension $L_{\infty, k}$ over $k_{\infty}$. Extend $\gamma$ to $\tilde{\gamma} \in$ $\operatorname{Gal}\left(L_{\infty, k} / k\right)$. Let $x \in X_{k}$. Then $\gamma$ acts on $x$ by $x^{\gamma}=\tilde{\gamma} x \tilde{\gamma}^{-1}$. Since $\operatorname{Gal}\left(L_{\infty, k} / k_{\infty}\right)$ is abelian, $x^{\gamma}$ is well-defined. In some cases, we will use the additive notation $\gamma x$ instead of the multiplicative one $x^{\gamma}$. We make $X_{k}$ into a $\Lambda=\mathbb{Z}_{p}[[T]]$-module in the following way;

$$
(1+T) x=\gamma x
$$

Iwasawa proved the following theorem. The idea to prove the theorem is to make $X_{k}$ into a $\Lambda$-module.

Theorem 3 (L. Washington [14, page 67]). Let $k_{\infty} / k$ be a $\mathbb{Z}_{p}$-extension. Let $p^{e_{n}}$ be the exact power of $p$ dividing the class number of $k_{n}$. Then there exist integers $\lambda \geq 0, \mu \geq 0$, and $\nu$, all independent of $n$, and an integer $n_{0}$, such that

$$
e_{n}=\lambda n+\mu p^{n}+\nu
$$

for all $n \geq n_{0}$. 
Let $\mathbb{Q}_{\infty} / \mathbb{Q}$ be the unique $\mathbb{Z}_{p}$-extension of $\mathbb{Q}$. Then the compositum $k \mathbb{Q}_{\infty}$ is a $\mathbb{Z}_{p}$-extension of $k$, which is said to be the basic $\mathbb{Z}_{p}$-extension of $k$. Ferrero and Washington [2] proved that the $\mu$-invariant is zero for the basic $\mathbb{Z}_{p}$-extension $k_{\infty} / k$ when $k$ is abelian over $\mathbb{Q}$. Iwasawa [7] constructed a non-basic $\mathbb{Z}_{p}$-extension whose $\mu$-invariant is not zero. It has been conjectured that we always have $\mu=0$ for the basic $\mathbb{Z}_{p}$-extension.

Two $\Lambda$-modules $M$ and $M^{\prime}$ are pseudo-isomorphic, written $M \sim M^{\prime}$, if there is a $\Lambda$-module map between them with finite kernel and cokernel. The relation $\sim$ is not reflexive in general. However, it can be shown that it is reflexive for finitely generated $\Lambda$-torsion modules. A non-constant polynomial $g(T) \in \Lambda$ is called distinguished if

$$
g(T)=T^{n}+a_{n-1} T^{n-1}+\cdots+a_{0}, p \mid a_{i}, 0 \leq i \leq n-1 .
$$

By the structure theorem of $\Lambda$-modules, every finitely generated $\Lambda$-module $M$ is pseudo-isomorphic to a module of the form

$$
\Lambda^{r} \oplus\left(\bigoplus_{i=1}^{s} \Lambda / p^{n_{i}}\right) \oplus\left(\bigoplus_{j=1}^{t} \Lambda / f_{j}^{m_{j}}(T)\right)
$$

where $r, s, t, n_{i}, m_{j} \in \mathbb{Z}$, and $f_{j}$ is distinguished and irreducible. The characteristic ideal $\left(\prod f_{j}^{m_{j}}\right)\left(\prod p^{n_{i}}\right) \Lambda$ is an invariant of $M$, which we will denote by $\operatorname{char}(M)$. Define the $\mu$-invariant of $M$ by $\mu=\sum_{i=1}^{s} n_{i}$, and the $\lambda$-invariant of $M$ by $\sum_{j=1}^{t} m_{j} \operatorname{deg}\left(f_{j}\right)$.

Theorem 4. Suppose $k_{\infty} / k$ is a $\mathbb{Z}_{p}$-extension and assume $\mu=0$. Then

$$
\left.X_{k} \simeq \mathbb{Z}_{p}^{\lambda} \oplus \text { ( finite p group }\right)
$$

as a $\mathbb{Z}_{p}$-module.

Proof. See Washington [14, page 286].

Let $k$ be a totally real number field. Fix a rational odd prime $p$, and for every integer $n \geq 0$, let $K_{n}=k\left(\zeta_{p^{n+1}}\right), K_{\infty}=\bigcup K_{n}$, where $\zeta_{p^{n}}$ is a $p^{n}$-th root of unity. Put $\Delta=\operatorname{Gal}\left(K_{0} / k\right)$ and $\Gamma=\operatorname{Gal}\left(K_{\infty} / K_{0}\right) \simeq \mathbb{Z}_{p}$ then $\operatorname{Gal}\left(K_{\infty} / k\right)=\Delta \times \Gamma$. Let $A_{n}$ be the Sylow $p$-subgroup of the ideal class group of $K_{n}$, and $Y_{n}$ be the Galois group $M_{n} / K_{n}$, where $M_{n}$ is the maximal abelian $p$-extension of $K_{n}$ unramified outside primes above $p$. Define

$$
\begin{gathered}
X_{k\left(\zeta_{p}\right)}=\lim _{\leftarrow} A_{n}, \\
Y_{k\left(\zeta_{p}\right)}=\lim _{\leftarrow} Y_{n}, \\
A_{\infty}=\lim _{\rightarrow} A_{n},
\end{gathered}
$$

all inverse limits with respect to the norm maps, the direct limit with respect to the induced map of lifting of ideals. The Iwasawa module $X_{k\left(\zeta_{p}\right)}$ is isomorphic to the Galois group of the maximal unramified abelian $p$-extension of $K_{\infty}$ over $K_{\infty}$ and $Y_{k\left(\zeta_{p}\right)} \simeq \operatorname{Gal}\left(M_{\infty} / K_{\infty}\right)$, where $M_{\infty}$ is the maximal abelian $p$-extension of $K_{\infty}$ unramified outside primes above $p$.

Define the Iwasawa algebra

$$
\mathbb{Z}_{p}[[\Gamma]]=\lim _{\leftarrow} \mathbb{Z}_{p}\left[\operatorname{Gal}\left(K_{n} / K_{0}\right)\right] .
$$


Fix a topological generator $\gamma_{0}$ of $\Gamma$. We identify $\mathbb{Z}_{p}[[\Gamma]]$ with formal power series ring $\Lambda=\mathbb{Z}_{p}[[T]]$ by $\gamma_{0} \rightarrow 1+T$. Write $\theta$ for the character with values in $\mathbb{Z}_{p}^{\times}$giving the action of $\Delta$ on $\zeta_{p}$. Let $\kappa$ be the character giving the action of $\Gamma$ on the group of $p$-power roots of unity. Put

$$
u=\kappa\left(\gamma_{0}\right) .
$$

For any integer $i=0,1, \ldots,|\Delta|-1$, define $\theta^{i}$-idempotent

$$
e_{i}=\frac{1}{|\Delta|} \sum_{\delta \in \Delta} \theta^{-i}(\delta) \delta
$$

The Iwasawa module $Y_{k\left(\zeta_{p}\right)}$ is a finitely generated $\Lambda$-module and $X_{k\left(\zeta_{p}\right)}$ is a finitely generated torsion $\Lambda$-module.

For every odd integer $i$, there exists a fraction of power series $G\left(T, \theta^{i}\right)$ in the field of fractions of $\Lambda$ satisfying

$$
G\left(u^{s}-1, \theta^{i}\right)=L_{p}\left(\theta^{1-i}, s\right),
$$

where $L_{p}\left(\theta^{1-i}, s\right)$ is the $p$-adic $L$-function of $\theta^{1-i}$. Hence $G\left(T, \theta^{i}\right)$ is characterized by the following relation:

$$
G\left(u^{s}-1, \theta^{i}\right)=L_{k}\left(\theta^{-i+s}, s\right) \prod_{\mathfrak{p} \mid p}\left(1-\theta^{-i+s}(\mathfrak{p}) N \mathfrak{p}^{-s}\right)
$$

for every negative integer $s$. For every odd integer $i$, let

$$
H\left(T, \theta^{i}\right)=\left\{\begin{array}{rll}
G\left(T, \theta^{i}\right), & i \neq 1 \quad \bmod |\Delta|, \\
(1+T-u) G(T, \theta), & i \equiv 1 & \bmod |\Delta|
\end{array}\right.
$$

Let

$$
\tau=\lim _{\leftarrow} \mu_{p^{n}} .
$$

By Kummer theory, we can prove that

$$
e_{1-i} Y_{k\left(\zeta_{p}\right)}(-1) \stackrel{\text { def }}{=} e_{1-i} Y_{k\left(\zeta_{p}\right)} \otimes_{\mathbb{Z}_{p}} \operatorname{Hom}_{\mathbb{Z}_{p}}\left(\tau, \mathbb{Z}_{p}\right) \simeq H o m\left(e_{i} A_{\infty}, \mathbb{Q}_{p} / \mathbb{Z}_{p}\right)
$$

Let $G_{i}(T)$ be a power series such that $G_{i}\left((1+T)^{-1}-1\right)$ is a characteristic power series of $\operatorname{Hom}\left(e_{i} A_{\infty}, \mathbb{Q}_{p} / \mathbb{Z}_{p}\right)$. The following theorem is proved by Wiles [15](the "Main Conjecture").

Theorem 5. For each odd integer $i, H\left(T, \theta^{i}\right) \Lambda=G_{i}(T) \Lambda$.

Let $\operatorname{char}\left(e_{i} X_{k\left(\zeta_{p}\right)}\right)=F_{i}(T) \Lambda$. By Iwasawa [6], $\operatorname{char}\left(\operatorname{Hom}\left(e_{i} A_{\infty}, \mathbb{Q}_{p} / \mathbb{Z}_{p}\right)\right)=$ $\operatorname{char}\left(e_{i} X_{k\left(\zeta_{p}\right)}\right)$. Hence we have the following equivalent form of the Main Conjecture.

Theorem 6. For each odd integer $i, F_{i}\left((1+T)^{-1}-1\right) \Lambda=H\left(T, \theta^{i}\right) \Lambda$.

\section{Proof of theorems}

Notations are the same as in section 1 . We define the minus-part of $X_{k\left(\zeta_{p}\right)}$ by

$$
X_{k\left(\zeta_{p}\right)}^{-}=\sum_{i=1 \text { odd }}^{|\Delta|} e_{i} X_{k\left(\zeta_{p}\right)} .
$$

We state the main theorem of this chapter. 
Theorem 7 (= Theorem 1). Let $S$ be a finite set of primes. Let $k$ be a totally real number field. Suppose we know $X_{k\left(\zeta_{p}\right)}^{-}$as a $\Lambda[\Delta]$-module up to pseudo-isomorphism for all $p \notin S$; then we can determine the zeta function $\zeta_{k}$ of $k$.

We let $\operatorname{ord}_{p}$ denote the usual valuation on $\overline{\mathbb{Q}_{p}}$, normalized by $\operatorname{ord}_{p}(p)=1$, and let $|x|=p^{-\operatorname{ord}_{p}(x)}$.

Lemma 1. Let $\left\{x_{n}\right\}$ be a sequence in $\mathbb{C}_{p}$, which converges to $x_{0} \neq 0$. Then $\operatorname{ord}_{p}\left(x_{n}\right)=\operatorname{ord}_{p}\left(x_{0}\right)$ for $n$ sufficiently large.

Proof. Since $x_{n}$ approaches $x_{0},\left|x_{n}-x_{0}\right|$ is strictly less than $\left|x_{0}\right|$ for $n$ sufficiently large. Therefore $\left|x_{n}\right|=\max \left\{\left|x_{n}-x_{0}\right|,\left|x_{0}\right|\right\}=\left|x_{0}\right|$ for $n$ sufficiently large.

Let $\delta_{i}=\# \operatorname{Gal}\left(k\left(\zeta_{p_{i}}\right) / k\right)$ for an odd prime $p_{i}$. Then $\delta_{i}$ is an even integer since $k$ is a totally real number field. When $p=2, \Delta=\operatorname{Gal}\left(k\left(\zeta_{4}\right) / k\right)$ so that $\delta=2$. Let $S$ be a finite set of primes which contains the prime number 2 .

Proposition 1. The Iwasawa modules $X_{k\left(\zeta_{p}\right)}^{-}$, for all primes not in $S$, determine the absolute value of $\zeta_{k}$ at negative integers, up to primes in $S$.

Proof. If $n$ is a negative even integer, then $\zeta_{k}(n)=0$. Fix a negative odd integer $n$. Let $p$ be a prime number not in $S$. Then $n \equiv i_{n} \bmod |\Delta|$, for some odd integer $i_{n}, 0 \leq i_{n} \leq|\Delta|-1$. It is well-known that the values $\zeta_{k}(n)$ are in $\mathbb{Q}$. By Theorem 6 , we know the value

$$
\begin{aligned}
\operatorname{ord}_{p}\left(G\left(u^{n}-1, \theta^{i_{n}}\right)\right) & =\operatorname{ord}_{p} L_{k}\left(\theta^{-i_{n}+n}, n\right) \prod_{\mathfrak{p} \mid p}\left(1-\theta^{-i_{n}+n}(\mathfrak{p}) N \mathfrak{p}^{-n}\right) \\
& =\operatorname{ord}_{p} L_{k}(1, n)=\operatorname{ord}_{p} \zeta_{k}(n) .
\end{aligned}
$$

Hence the absolute value of $\zeta_{k}(n)$ is determined up to primes in $S$.

Remark. By definition, the $p$-adic $L$-function $L_{p}\left(\theta^{i}, s\right)$ of $\theta^{i}$ is the continuous function from $\mathbb{Z}_{p} \backslash\{1\}$ to $\mathbb{C}_{p}$ satisfying $L_{p}\left(\theta^{i}, s\right)=L_{k}\left(\theta^{i}, s\right) \prod_{\mathfrak{p} \mid p}\left(1-\theta^{i}(\mathfrak{p}) N \mathfrak{p}^{-s}\right)$ for all rational integers $s \leq 0$ with $s \equiv 1 \bmod \delta$, where $\delta=\# \operatorname{Gal}\left(k\left(\zeta_{p}\right) / k\right)$, for an odd integer $p$. For all integers $i$ and $n>1, L_{k}\left(\theta^{i}, 1-n\right)$ is non-zero if and only if $i$ and $n$ have the same parity.

Let $\sigma_{i}=p_{i}-1$ for an odd prime $p_{i}$, and $\sigma_{i}=2$ if $p_{i}=2$. Then $\delta_{i}$ divides $\sigma_{i}$.

Proposition 2. Let $S=\left\{p_{1}, \ldots, p_{t}\right\}$ be any finite set of primes. Then there is a sequence $\left\{a_{n}\right\}$ of odd integers such that ord ${ }_{p}\left(\zeta_{k}\left(a_{n}\right)\right)$ is constant for $n$ sufficiently large for all primes $p$ in $S$.

Proof. Let $a_{n}=1-2 \sigma_{1} \cdots \sigma_{t}-2 \sigma_{1} \cdots \sigma_{t} p_{1}^{n} \cdots p_{t}^{n}$; then

$$
L_{p_{i}}\left(1, a_{n}\right)=\left(\prod_{\mathfrak{p} \mid p_{i}}\left(1-N \mathfrak{p}^{-a_{n}}\right)\right) \zeta_{k}\left(a_{n}\right),
$$

so we know that $\zeta_{k}\left(a_{n}\right)$ approaches $L_{p_{i}}\left(1,1-2 \sigma_{1} \cdots \sigma_{t}\right) p_{i}$-adically with $n$. By the remark above, $L_{p_{i}}\left(1,1-2 \sigma_{i} \cdots \sigma_{t}\right) \neq 0$. Therefore there exists a positive integer $N$ such that $\operatorname{ord}_{p_{i}} \zeta_{k}\left(a_{n}\right)=\operatorname{ord}_{p_{i}} \zeta_{k}\left(1-2 \sigma_{1} \cdots \sigma_{t}\right)$ for every integer $n>N$, and $i=1, \cdots, t$. This completes the proof.

By the functional equation, we have the following equation.

$$
A^{s} \Gamma(s / 2)^{N} \zeta_{k}(s)=A^{1-s} \Gamma((1-s) / 2)^{N} \zeta_{k}(1-s),
$$


where $A=d_{k}^{1 / 2} \pi^{-N / 2}, N=[k: \mathbb{Q}]$, and $d_{k}$ is the absolute value of the discriminant of $k$. Hence we have

$$
\begin{aligned}
\zeta_{k}(1-s) & =A^{2 s-1} \Gamma(s / 2)^{N} \Gamma((1-s) / 2)^{-N} \zeta_{k}(s) \\
& =A^{2 s-1}(\Gamma(s / 2) / \Gamma((1-s) / 2))^{N} \zeta_{k}(s) \\
& =A^{2 s-1}\left(\Gamma(s) 2^{1-s} \pi^{-1 / 2} \cos ((s \pi) / 2)\right)^{N} \zeta_{k}(s) .
\end{aligned}
$$

Finally, we get the following equation.

$$
\left|\zeta_{k}(1-\ell)\right|=A^{2 \ell-1} \Gamma(\ell)^{N}\left(2^{1-\ell}\right)^{N} \pi^{-N / 2}\left|\zeta_{k}(\ell)\right|
$$

for any positive even integer $\ell$.

Now we are ready to prove Theorem 7 by following the idea of Goss and Sinnott [4]. Let $n$ be a rational number, $S$ be a finite set of primes. We define $(n)_{S-p a r t}=\prod_{p \in S} p^{\operatorname{ord}_{p}(n)}$, and $(n)_{n o n-S-\text { part }}=n /(n)_{S-\text { part }}$. Let $x>0$ be a real number. Then from the equation (2), we have the following equation;

$$
\left|\zeta_{k}(1-\ell)\right| / \Gamma(\ell)^{x}=\left(A^{2} 2^{-N}\right)^{\ell} \Gamma(\ell)^{N-x} 2^{N} \pi^{-N / 2} A^{-1}\left|\zeta_{k}(\ell)\right|
$$

By Stirling's formula,

$$
B^{s} / \Gamma(s) \rightarrow 0 \text { as } s \rightarrow \infty
$$

for any real $B>0$. Moreover, $\zeta_{k}(\ell) \rightarrow 1$ as $\ell \rightarrow \infty$. Choose a sequence $\left\{a_{n}\right\}$ as in Proposition 2, and let $a_{n}=1-\ell_{n}$. By Propositions 1 and 2 , we know the value of

$$
\left|\zeta_{k}\left(1-\ell_{n}\right)\right| / \Gamma\left(\ell_{n}\right)^{x}
$$

up to an (unknown) constant independent of $n$, as long as $n$ is sufficiently large. The right-hand side of the equation (3) approaches 0 as $\ell$ goes to $\infty$ if $N<x$, and goes to $\infty$ if $N>x$. Hence the same is true of (4). Hence we can read off $N$. Going back to the equation (2) with $\ell=\ell_{n}$, we can determine $A$;

$$
\begin{aligned}
A & =\lim _{n \rightarrow \infty} \exp \left[\left[1 /\left(2 \ell_{n}-1\right)\right] \log \left[\frac{\left|\zeta_{k}\left(1-\ell_{n}\right)\right|_{\text {non-S-part }}\left|\zeta_{k}\left(1-\ell_{n}\right)\right|_{S-\text { part }}}{\Gamma\left(\ell_{n}\right)^{N}\left(2^{1-\ell_{n}}\right)^{N} \pi^{-N / 2}\left|\zeta_{k}\left(\ell_{n}\right)\right|}\right]\right] \\
& =\lim _{n \rightarrow \infty} \exp \left[\left[1 /\left(2 \ell_{n}-1\right)\right] \log \left[\frac{\left|\zeta_{k}\left(1-\ell_{n}\right)\right|_{\text {non-S-part }}}{\Gamma\left(\ell_{n}\right)^{N}\left(2^{1-\ell_{n}}\right)^{N} \pi^{-N / 2}\left|\zeta_{k}\left(\ell_{n}\right)\right|}\right]\right]
\end{aligned}
$$

by Propositions 1 and 2 . Hence we know the discriminant $d_{k}$. Here $1-a_{n}$ is a multiple of 4 since $\sigma_{i}$ is even. Since the value $\cos (4 m \pi / 2)$ for integer $m$ and the values of zeta function at positive integers not equal to 1 are positive, we know, by the equation (1), the values $\zeta_{k}\left(a_{n}\right)$ are positive. By Proposition 1, we know the non- $S$-part of the values of zeta function at $a_{n}$, and by Proposition 2, the $S$-part is constant for $n$ sufficiently large. Hence, with the functional equation, we can determine the $S$-part of the values of the zeta function at the sequence $a_{n}$ for large $n$, i.e, we have:

$$
\begin{aligned}
\zeta_{k}\left(1-\ell_{n}\right)_{S-\text { part }} & =\lim _{m \rightarrow \infty} \frac{A^{2 \ell_{m}-1}\left(\Gamma\left(\ell_{m}\right) 2^{1-\ell_{m}} \pi^{-1 / 2} \cos \left(\left(\ell_{m} \pi\right) / 2\right)\right)^{N} \zeta_{k}\left(\ell_{m}\right)}{\zeta_{k}\left(1-\ell_{m}\right)_{n o n-S-p a r t}} \\
& =\lim _{m \rightarrow \infty} \frac{A^{2 \ell_{m}-1}\left(\Gamma\left(\ell_{m}\right) 2^{1-\ell_{m}} \pi^{-1 / 2}\right)^{N}}{\zeta_{k}\left(1-\ell_{m}\right)_{n o n-S-p a r t}}
\end{aligned}
$$

for $n$ sufficiently large. Therefore, by Proposition 1 , we know the values $\zeta_{k}\left(1-\ell_{n}\right)$ for $n$ sufficiently large. 
Let

$$
\zeta_{k}(s)=\sum b_{n} / n^{s}
$$

Then we have

$$
\sum_{m=1}^{\infty} b_{m} / m^{\ell_{n}}=A^{2\left(1-\ell_{n}\right)-1} \Gamma\left(\left(1-\ell_{n}\right) / 2\right)^{N} \Gamma\left(\left(\ell_{n}\right) / 2\right)^{-N} \zeta_{k}\left(1-\ell_{n}\right) .
$$

We know the values of the right-hand side of the above equation for $n$ sufficiently large, which will be denoted by $c_{n}$. We know $b_{1}=1$, and

$$
b_{2}=\lim _{n \rightarrow \infty}\left(c_{n}-1\right) 2^{\ell_{n}} .
$$

Continuing the above process, we can determine all the coefficients $b_{m}$ 's, so we can determine the zeta function $\zeta_{k}(s)$. This completes the proof of Theorem 7 .

Let $k, k^{\prime}$ be totally real number fields, and let $S$ be a finite set of primes containing all the primes which are ramified in $k$ and $k^{\prime}$. Then the number fields $k$ and $k^{\prime}$ are linearly disjoint with $\mathbb{Q}\left(\mu_{p^{\infty}}\right)$ over $\mathbb{Q}$ for $p \notin S$. Let $K_{\infty}=k\left(\mu_{p \infty}\right)$, and let $K_{\infty}^{\prime}=k^{\prime}\left(\mu_{p^{\infty}}\right)$. Then we may identify $\operatorname{Gal}\left(K_{\infty} / k\right)$ and $\operatorname{Gal}\left(K_{\infty}^{\prime} / k^{\prime}\right)$ (they are both naturally isomorphic to $\operatorname{Gal}\left(\mathbb{Q}\left(\mu_{p^{\infty}}\right) / \mathbb{Q}\right)$, so that we may compare the Iwasawa

modules $X_{k\left(\zeta_{p}\right)}^{-}$and $X_{k^{\prime}\left(\zeta_{p}\right)}^{-}$as $\Lambda[\Delta]$-modules. Then, from Theorem 7 , we have the following corollary.

Corollary 1. Let $k$ and $k^{\prime}$ be totally real number fields. Let $S$ be a finite set of primes containing all the primes which are ramified in $k$ and $k^{\prime}$. Assume that the two Iwasawa modules

$$
X_{k\left(\zeta_{p}\right)}^{-} \sim X_{k^{\prime}\left(\zeta_{p}\right)}^{-}
$$

are pseudo-isomorphic as $\Lambda[\Delta]$-modules for all $p \notin S$; then

$$
\zeta_{k}=\zeta_{k^{\prime}} .
$$

\section{Arithmetic equivalence}

Let $k$ be a number field, and $\mathfrak{o}_{k}$ be its ring of integers. Let $p \mathfrak{o}_{k}=\mathfrak{p}_{g}^{e_{1}} \cdots \mathfrak{p}_{g}^{e_{g}}$ be the decomposition of a prime number $p \in \mathbb{Z}$, let $f_{i}=\left[\left(\mathfrak{o}_{k} / \mathfrak{p}_{i}\right): \mathbb{Z} / p\right]$ be the degree of $\mathfrak{p}_{i}$, and $e_{i}$ be the ramification indices, numbered so that $f_{i} \leq f_{i+1}$. Then the tuple $A=\left(f_{1}, \ldots, f_{g}\right)$ is called the splitting type of $p$ in $k$. We define a set $P_{k}(A)$ by $P_{k}(A)=\{p \in \mathbb{Z}: p$ has splitting type $A$ in $k\}$. The notation $S \doteq T$ will be used to indicate that these two sets differ by at most a finite number of elements. Two subgroups $H, H^{\prime}$ of a finite group $G$ are said to be Gassmann equivalent in $G$ when

$$
\left|c^{G} \cap H\right|=\left|c^{G} \cap H^{\prime}\right|
$$

for every conjugacy class $c^{G}=\left\{g c g^{-1} \mid g \in G\right\}$ in $G$ and $c$ in $G$. Let $k$ and $k^{\prime}$ be number fields, and $L$ be a Galois extension of $\mathbb{Q}$ containing $k$ and $k^{\prime}$. Write $H=\operatorname{Gal}(L / k), H^{\prime}=\operatorname{Gal}\left(L / k^{\prime}\right)$ and $G=\operatorname{Gal}(L / \mathbb{Q})$. The normal core of $k$ is the largest subfield of $k$ normal over $\mathbb{Q}$. It is the fixed field of the subgroup $\left\langle H^{\sigma} \mid \sigma \in \operatorname{Gal}(L / \mathbb{Q})\right\rangle$ generated by all conjugates of $H$. We call $k, k^{\prime}$ arithmetically equivalent if $H$ and $H^{\prime}$ are Gassmann equivalent in $G$. Note that this definition is independent of the choice of $L$ and that if $k, k^{\prime}$ are arithmetically equivalent, then they have the same normal closure.

Lemma 2 (Perlis [10]). Two arithmetically equivalent number fields $k$ and $k^{\prime}$ have the same normal core. 
With this notation we have the following theorem.

Theorem 8. The following are equivalent.

(a) $\zeta_{k}(s)=\zeta_{k^{\prime}}(s)$.

(b) $P_{k}(A)=P_{k^{\prime}}(A)$ for every tuple $A$.

(c) $P_{k}(A) \doteq P_{k^{\prime}}(A)$ for every tuple $A$.

(d) $H=\operatorname{Gal}(L / k)$ and $H^{\prime}=\operatorname{Gal}\left(L / k^{\prime}\right)$ are Gassmann equivalent in $G$.

(e) $\mathbb{Q}[H \backslash G]$ is isomorphic to $\mathbb{Q}\left[H^{\prime} \backslash G\right]$ as a $\mathbb{Q}[G]$-module.

Proof. See Komatsu [8].

Let $H$ and $H^{\prime}$ be Gassmann equivalent. Let $\left\{\rho_{1}, \cdots, \rho_{t}\right\}$ and $\left\{\rho_{1}^{\prime}, \cdots, \rho_{t}^{\prime}\right\}$ be right coset representatives of $H \backslash G$ and $H^{\prime} \backslash G$, respectively. Then we have two homomorphisms $\pi, \pi^{\prime}$ from $G$ into symmetric group $S_{t}$ given by $\pi_{g}(i)=j$, where $H \rho_{i} g=H \rho_{j}$, and $\pi_{g}^{\prime}(i)=j$, where $H^{\prime} \rho_{i}^{\prime} g=H^{\prime} \rho_{j}^{\prime}$. Let $D$ and $D^{\prime}$ be the linear representations of $G$ induced from the unit representations of $H$ and $H^{\prime}$. Their characters $\chi, \chi^{\prime}$ are given by

$$
\begin{gathered}
\chi(g)=\left|g^{G} \cap H\right|\left|C_{G}(g)\right| /|H|, \\
\chi^{\prime}(g)=\left|g^{G} \cap H^{\prime}\right|\left|C_{G}(g)\right| /\left|H^{\prime}\right|,
\end{gathered}
$$

for $g \in G$, where $C_{G}(g)$ is the centralizer. By Theorem $8, \chi=\chi^{\prime}$ so that the representations $D, D^{\prime}: G \rightarrow G L_{t}(\mathbb{Q})$ are isomorphic. Thus there is a rational $t \times t$ matrix $M \in G L_{t}(\mathbb{Q})$ satisfying the following relation :

$$
D(g) M=M D^{\prime}(g)
$$

for every $g \in G$. By clearing the denominators, we may assume that $M$ is in $G L_{t}(\mathbb{Z})$. A matrix $M=\left(m_{i j}\right)$ satisfies the above equation if and only if $m_{i j}=m_{\pi_{g}(i), \pi_{g}^{\prime}(j)}$ for all $g \in G$. With the same notation as in Theorem 8, we have the following proposition.

Proposition 3. Let $k$ and $k^{\prime}$ be arithmetically equivalent fields. Then there is an exact sequence of right $\mathbb{Z}_{p}[G]$-modules

$$
0 \rightarrow \mathbb{Z}_{p}[H \backslash G] \rightarrow \mathbb{Z}_{p}\left[H^{\prime} \backslash G\right] \rightarrow A \rightarrow 0,
$$

where $A$ is a finite right- $\mathbb{Z}_{p}[G]$-module.

Proof. Let $M$ be a matrix satisfying the condition

$$
m_{i j}=m_{\pi_{g}(i), \pi_{g}^{\prime}(j)} .
$$

Define a map $\varphi$ from $\mathbb{Z}_{p}[H \backslash G] \rightarrow \mathbb{Z}_{p}\left[H^{\prime} \backslash G\right]$ by

$$
\varphi\left(H \rho_{i}\right)=m_{i 1} H^{\prime} \rho_{1}^{\prime}+\cdots+m_{i t} H^{\prime} \rho_{t}^{\prime}, i=1, \cdots, t,
$$

so $\varphi$ may be represented by a matrix $M$ with a basis $\left\{\rho_{1}, \cdots, \rho_{t}\right\}$ and $\left\{\rho_{1}^{\prime}, \cdots, \rho_{t}^{\prime}\right\}$. By the equation (5), $\varphi$ is a right $-\mathbb{Z}_{p}[G]$-module homomorphism. Since $M$ is invertible, $\varphi$ is injective. Moreover, we have the following equation.

$$
\operatorname{det} M\left(\begin{array}{c}
H^{\prime} \rho_{1}^{\prime} \\
\vdots \\
H^{\prime} \rho_{t}^{\prime}
\end{array}\right)=(\operatorname{det} M) M^{-1}\left(\begin{array}{c}
\varphi\left(H \rho_{1}\right) \\
\vdots \\
\varphi\left(H \rho_{t}\right)
\end{array}\right)
$$


Hence cokernel $\varphi$ is killed by $\operatorname{det} M$, but cokernel $\varphi$ is a finitely generated $\mathbb{Z}_{p^{-}}$ module. Therefore cokernel $\varphi$ is finite. This completes the proof.

Remark. If $p$ does not divide the order of $H$, then we can take $A$ to be zero. In the case, both $\mathbb{Z}_{p}[H \backslash G]$ and $\mathbb{Z}_{p}\left[H^{\prime} \backslash G\right]$ are projective $\mathbb{Z}_{p}[G]$-modules. A projective module is determined by its character $\chi$; hence, they are isomorphic. For details, see Komatsu [8].

Write

$$
\Lambda_{t}=\mathbb{Z}_{p}\left[\left[(1+T)^{p^{t}}-1\right]\right],
$$

where $\Lambda_{0}=\Lambda=\mathbb{Z}_{p}[[T]]$. For the rest of this paper, $p$ is a fixed prime number, and let $L$ be a normal closure of $k$ and $k^{\prime}$. Let $L_{0} \subset L_{1} \subset L_{2} \subset \cdots \subset L_{\infty}$ be the basic $\mathbb{Z}_{p}$-extension over the field $L=L_{0}$. Put $\Gamma=\operatorname{Gal}\left(L_{\infty} / L\right) \simeq \mathbb{Z}_{p}$. When $p$ does not divide $[L: \mathbb{Q}]$, we can identify the following Galois groups $\operatorname{Gal}\left(k_{\infty} / k\right), \operatorname{Gal}\left(k_{\infty}^{\prime} / k^{\prime}\right)$ and $\operatorname{Gal}\left(L_{\infty} / L\right)$. Komatsu proved that two Iwasawa modules $X_{k}$ and $X_{k^{\prime}}$ are isomorphic as $\mathbb{Z}_{p}[[\Gamma]]=\Lambda_{L}$-modules when $p$ does not divide $[L: Q]$. Let $\Lambda_{k}=\mathbb{Z}_{p}\left[\left[\operatorname{Gal}\left(k_{\infty} / k\right)\right]\right]$. Now for any prime $p$ including the above exceptional case, regarding $\Lambda_{L}$ as a subring of $\Lambda_{k}$, we have $\Lambda_{L}=\Lambda_{k, t}$ for some $t \geq 0$. In this chapter, we will prove that the Iwasawa modules $X_{k, \lambda}$ and $X_{k^{\prime}, \lambda}$ are pseudo-isomorphic as $\Lambda_{L}$-modules for any prime $p$.

\section{Proof of theorems}

Let $k$ be a number field, and let $L$ be the Galois closure of $k$ over $\mathbb{Q}$. In addition, we assume that $L \cap k_{\infty}=k$. Write $\operatorname{Gal}(L / k)=H$. Since $L \cap k_{\infty}=k$, the group $H$ can be considered as $\operatorname{Gal}\left(L_{n} / k_{n}\right)$ for any $n \geq 0$, and it commutes with $\Gamma$. Hence the group $H$ acts on $X_{L}$. Regard $\Lambda_{L}$ as a subring of $\Lambda_{k}$, so that $\Lambda_{L}$ acts on $X_{k}$. Recall that $X_{\lambda}=X /\left(\mathbb{Z}_{p}-\right.$ torsion $\left.(X)\right)$ for a $\Lambda$-module $X$.

Proposition 4. The Iwasawa modules $X_{L, \lambda}^{H}$ and $X_{k, \lambda}$ are pseudo-isomorphic as $\Lambda_{L}$-modules.

Proof. Let $|H|=[L: k]=p^{\alpha} m$, where $(m, p)=1$. For each $n$, we choose $c_{n}|H| \equiv$ $p^{\alpha} \bmod p^{t_{n}}$, so that $p^{t_{n}}$ exceeds the order of $A_{n, L}$ and $A_{n, k}$, where $A_{n, M}$ is the $p$-Sylow subgroup of the ideal class group of the $n$-th layer of the basic $\mathbb{Z}_{p^{-}}$-extension over a number field $M$. Let $i$ be the lifting map from $A_{n, k}$ to $A_{n, L}^{H}$, and $N$ be the norm map on ideal classes. Let $\beta_{n}$ be an element of the kernel of the map $i$. Then

$$
0=c_{n} N \circ i\left(\beta_{n}\right)=p^{\alpha} \beta_{n},
$$

so that the kernel of $i$ is killed by $p^{\alpha}$ for any $n$. Let $\gamma_{n}$ be in $A_{n, L}^{H}$. We have the following equation:

$$
i\left(c_{n} N \gamma_{n}\right)=i\left(c_{n}|H|\right) \gamma_{n}=p^{\alpha} \gamma_{n},
$$

so that the cokernel of $i$ is killed by $p^{\alpha}$ for any $n$. The lifting map $i$ commutes with the inverse limit, and the map $i: \lim _{\leftarrow} A_{n, k} \longrightarrow \lim _{\leftarrow} A_{n . L}^{H}$ is a $\Lambda_{L}$-homomorphism since $H$ and $\Gamma$ commute with each other. Define the induced map $i^{*}$ of $i$ from $X_{k, \lambda}$ to $X_{L, \lambda}^{H}$ by $i^{*}(\bar{x})=\overline{i(x)}$, where $\bar{x}$ is the reduction map from $X$ to $X_{\lambda}$. The map $i^{*}$ is well-defined since the image of the $\mathbb{Z}_{p}$-torsion of $X_{k}$ is contained in the $\mathbb{Z}_{p}$-torsion of $X_{L}^{H}$. The map $i^{*}$ is injective: if $i^{*}(\bar{x})=0$, then $p^{m} i(x)=0$ for some integer $m$; hence, by $(6), p^{t} x=0$ for some integer $t$, which means that $x$ is in the $\mathbb{Z}_{p^{-}}$torsion of $X_{k}$ i.e. $x \equiv 0$ in $X_{k, \lambda}$. Let $\bar{y}$ be any element of $X_{L, \lambda}^{H}$. Then, by the above formula 
(7), $p^{\alpha} \bar{y}=\overline{p^{\alpha} y}=\overline{i(x)}=i^{*}(\bar{x})$ for some $\bar{x}$ in $X_{k, \lambda}$. Since $X_{k, \lambda}$ and $X_{L, \lambda}^{H}$ are finitely generated $\mathbb{Z}_{p}$-modules, the induced map $i^{*}$ is a pseudo-isomorphism.

Lemma 3. Let $G$ be a group. For any prime number $p$, for any $\mathbb{Z}_{p}[G]$-module $A$, and a subgroup $H<G$,

$$
\operatorname{Hom}_{\mathbb{Z}_{p}[G]}\left(\mathbb{Z}_{p}[H \backslash G], A\right) \simeq A^{H},
$$

where $A^{H}$ is the subset of elements of $A$ fixed under $H$.

Proof. The isomorphism is given by

$$
\phi \longrightarrow \phi(H e) \text { for } \phi \in H_{o m_{\mathbb{Z}_{p}[G]}}\left(\mathbb{Z}_{p}[H \backslash G], A\right) \text {. }
$$

Remark. Let $R$ be a ring, and assume that $A$ is also a $R$-module. Assume $R$ commutes with the action of $G$. Then $\operatorname{Hom}_{\mathbb{Z}_{p}[G]}\left(\mathbb{Z}_{p}[H \backslash G], A\right) \simeq A^{H}$ as a $R$-module by making $(r \phi)(x)=r(\phi(x))$. The basic idea of the proof of the main theorem of this section is due to the above lemma which is used in Perlis and Colwell [11].

Let $k$ and $k^{\prime}$ be two isomorphic number fields and $\phi$ be an automorphism of $\overline{\mathbb{Q}}$ such that $\phi(k)=k^{\prime}$. Let $\gamma$ be a topological generator of $\operatorname{Gal}\left(k_{\infty} / k\right)$. Then $\gamma^{\prime}=\phi \gamma \phi^{-1}$ is a topological generator of $\operatorname{Gal}\left(k_{\infty}^{\prime} / k^{\prime}\right)$. We make $X_{k}$ and $X_{k^{\prime}}$ into $\Lambda=\mathbb{Z}_{p}[[T]]$-modules in the following way.

$$
\gamma x=(1+T) x \text { and } \quad \gamma^{\prime} x^{\prime}=(1+T) x^{\prime},
$$

where $x \in X_{k}$ and $x^{\prime} \in X_{k^{\prime}}$.

Proposition 5. Let $k$ and $k^{\prime}$ be two isomorphic number fields. Then the Iwasawa modules $X_{k}$ and $X_{k^{\prime}}$ are isomorphic as $\Lambda$-modules for any prime number $p$.

Proof. Let $e$ be an integer such that $\mathbb{Q}_{\infty} \cap k=\mathbb{Q}_{e}$ and $k_{n}=k \mathbb{Q}_{n+e}$ be the $n$-th layer of the basic $\mathbb{Z}_{p}$-extension of $k$. Since $\mathbb{Q}_{n+e}$ is the normal extension of $\mathbb{Q}$, $\phi\left(k_{n}\right)=k_{n}^{\prime}$. Let $x=\left(x_{1}, \cdots, x_{n}, \cdots\right) \in X_{k}$. Let the fractional ideal $\mathfrak{a}_{n}$ be a representative of $x_{n}$. Define $\phi\left(x_{n}\right)$ to be the class of $\mathfrak{a}_{n}^{\phi}$. Then

$$
\begin{aligned}
N \gamma_{n}^{\prime} \circ \phi\left(x_{n}\right) & =\left(1+\gamma_{n}^{\prime}+\cdots+\gamma_{n}^{\prime p-1}\right) \phi\left(x_{n}\right) \\
& =\phi\left(1+\gamma_{n}+\cdots+\gamma_{n}^{p-1}\right)\left(x_{n}\right)=\phi \circ N \gamma_{n}\left(x_{n}\right) .
\end{aligned}
$$

Hence $\phi$ induces a map from $X_{k}$ to $X_{k^{\prime}}$ which is also denoted by $\phi$. Moreover, it is a $\Lambda$-module homomorphism;

$$
\begin{aligned}
T \cdot \phi(x) & =\left(\gamma^{\prime}-1\right) \phi(x)=\gamma^{\prime} \phi(x) / \phi(x) \\
& =\phi(\gamma x) / \phi(x)=\phi((\gamma-1) x)=\phi(T \cdot x) .
\end{aligned}
$$

The map $\phi$ is trivially bijective. This completes the proof.

Lemma 4 (Komatsu). Let $k$ and $k^{\prime}$ be number fields such that $\zeta_{k}=\zeta_{k^{\prime}}$. Let $K$ be a finite Galois extension of $\mathbb{Q}$. Then we have $\zeta_{k K}=\zeta_{k^{\prime} K}$.

Proof. See Komatsu [8] .

Let $L$ be the Galois closure of $k$ and $k^{\prime}$, and $L_{\infty} / L$ be the basic $\mathbb{Z}_{p}$-extension. Put $\Gamma=\operatorname{Gal}\left(L_{\infty} / L\right)$ and $\Lambda_{L}=\mathbb{Z}_{p}[[\Gamma]]$.

Now we restate the main theorem of this section. 
Theorem 9. Let $p$ be a prime number. Let $k$ and $k^{\prime}$ be number fields such that $\zeta_{k}=\zeta_{k^{\prime}}$. Then the Iwasawa modules

$$
X_{k, \lambda} \sim X_{k^{\prime}, \lambda}
$$

as $\Lambda_{L}=\Lambda_{k, t}$-modules for some integer $t \geq 0$.

Proof. Let $L$ be the Galois closure of $k$ and $k^{\prime}$. Let $e$ be an integer such that $k \cap \mathbb{Q}_{\infty}=\mathbb{Q}_{e}$. By Lemma $2, k^{\prime} \cap \mathbb{Q}_{\infty}=\mathbb{Q}_{e}$. Let $m$ be the largest integer such that $\mathbb{Q}_{m} \subset L$, where $\mathbb{Q}_{m}$ is the $m$-th layer of the basic $\mathbb{Z}_{p}$-extension $\mathbb{Q}_{\infty}$ of $\mathbb{Q}$. Put $k_{m}=k \mathbb{Q}_{m}$ and $k_{m}^{\prime}=k^{\prime} \mathbb{Q}_{m}$. By Lemma 4 ,

$$
\zeta_{k_{m}}=\zeta_{k_{m}^{\prime}} .
$$

Let $G=\operatorname{Gal}(L / \mathbb{Q}), K=\operatorname{Gal}\left(L / \mathbb{Q}_{m}\right), H=\operatorname{Gal}\left(L / k_{m}\right)$, and $H^{\prime}=\operatorname{Gal}\left(L / k_{m}^{\prime}\right)$. By the above equation (8) and Theorem 8, two subgroups $H$ and $H^{\prime}$ of $G$ are Gassmann equivalent in $G$. Hence we have an exact sequence by Proposition 3:

$$
0 \rightarrow \mathbb{Z}_{p}[H \backslash G] \rightarrow \mathbb{Z}_{p}\left[H^{\prime} \backslash G\right] \rightarrow A \rightarrow 0,
$$

where $A$ is a finite $\mathbb{Z}_{p}[G]$-module. Also note that $K$ is normal in $G$, and that $H$ and $H^{\prime}$ act on $X_{L}$. Since $L \cap \mathbb{Q}_{\infty}=\mathbb{Q}_{m}, K$ acts on $X_{L, \lambda}$ so that $X_{L, \lambda}$ is a right $\mathbb{Z}_{p}[K]$-module. Consider $\mathbb{Z}_{p}[G]$ as a left $\mathbb{Z}_{p}[K]$-module. Then we can form the tensor product:

$$
X^{\prime}=X_{L, \lambda} \otimes_{\mathbb{Z}_{p}[K]} \mathbb{Z}_{p}[G]
$$

Then $X^{\prime}$ is a right $\mathbb{Z}_{p}[G]$-module via the action of $\mathbb{Z}_{p}[G]$ on the second factor. We have an exact sequence from the equation (9).

$$
\begin{aligned}
0 & \rightarrow \operatorname{Hom}_{\mathbb{Z}_{p}[G]}\left(A, X^{\prime}\right) \\
& \rightarrow \operatorname{Hom}_{\mathbb{Z}_{p}[G]}\left(\mathbb{Z}_{p}\left[H^{\prime} \backslash G\right], X^{\prime}\right) \\
& \rightarrow \operatorname{Hom}_{\mathbb{Z}_{p}[G]}\left(\mathbb{Z}_{p}[H \backslash G], X^{\prime}\right) \\
& \rightarrow \operatorname{Ext}_{\mathbb{Z}_{p}[G]}^{1}\left(A, X^{\prime}\right) \rightarrow \cdots
\end{aligned}
$$

First, we will prove that

$$
\operatorname{Hom}_{\mathbb{Z}_{p}[G]}\left(\mathbb{Z}_{p}[H \backslash G], X^{\prime}\right) \sim \bigoplus_{p^{m}-\text { copies }} X_{k, \lambda}
$$

as a $\Lambda=\mathbb{Z}_{p}\left[\left[\operatorname{Gal}\left(L_{\infty} / L\right)\right]\right]$-module, where $p^{m}=[G: K]$. Let $\left\{\rho_{1}, \cdots, \rho_{p^{m}}\right\}$ be right coset representatives of $K \backslash G$ with $\rho_{1}=1$. Then

$$
X^{\prime} \simeq X_{L, \lambda} \otimes \rho_{1}+\cdots+X_{L, \lambda} \otimes \rho_{p^{m}}
$$

as a $\Lambda_{L}$-module. Note that this is a direct sum. Let $h \in H$. Since $h \in K$ and $K$ is normal in $G, \rho_{i} h \rho_{i}^{-1} \in K$ for any $\rho_{i} \in G$. Let $x \in X_{L, \lambda}$.

$$
\begin{aligned}
\left(x \otimes \rho_{i}\right) h & =x \otimes \rho_{i} h \\
& =x \otimes \rho_{i} h \rho_{i}^{-1} \rho_{i} \\
& =x^{\rho_{i} h \rho_{i}^{-1}} \otimes \rho_{i} \in X_{L, \lambda} \otimes \rho_{i} .
\end{aligned}
$$

Let $x_{1} \otimes \rho_{1}+\cdots+x_{p^{m}} \otimes \rho_{p^{m}} \in X^{\prime}, g \in G$ and $\gamma \in \Gamma$. Then $\rho_{i} g=k_{i} \rho_{\pi_{g}(i)}$ for some permutation $\pi_{g}$ on $\left\{1, \ldots, p^{m}\right\}$, where $k_{i} \in K$. Since $\gamma$ commutes with $k_{i}$, 
we have the following equation:

$$
\begin{aligned}
\left(\sum x_{i} \otimes \rho_{i}\right) g \gamma & =\left(\sum x_{i}^{k_{i}} \otimes \rho_{\pi_{g}(i)}\right) \gamma \\
& =\left(\sum x_{i}^{k_{i} \gamma} \otimes \rho_{\pi_{g}(i)}\right)=\left(\sum x_{i}^{\gamma k_{i}} \otimes \rho_{\pi_{g}(i)}\right) \\
& =\left(\sum x_{i}^{\gamma} \otimes \rho_{i}\right) g=\left(\sum x_{i} \otimes \rho_{i}\right) \gamma g .
\end{aligned}
$$

Therefore $\Lambda$ commute with the action of $G$ on $X^{\prime}$. By Lemma 3 , the remark below Lemma 3 , and the above equation (10), we have:

$$
H o m_{\mathbb{Z}_{p}[G]}\left(\mathbb{Z}_{p}[H \backslash G], X^{\prime}\right)=\left(X^{\prime}\right)^{H}=\sum\left(X_{L, \lambda} \otimes \rho_{i}\right)^{H} .
$$

We have a $\Lambda$-module isomorphism: $\phi: X_{L, \lambda} \otimes \rho_{i} \rightarrow X_{L, \lambda}$ by sending $x \otimes \rho_{i} \rightarrow x$. Again by (10),

$$
\sum\left(X_{L, \lambda} \otimes \rho_{i}\right)^{H} \simeq \sum X_{L, \lambda}^{\rho_{i} H \rho_{i}^{-1}}
$$

Since $H$ and $\rho_{i} H \rho_{i}^{-1}$ are conjugate in $G$, their fixed fields are isomorphic. By Propositions 4 and 5 , we have the following equation.

$$
H o m_{\mathbb{Z}_{p}[G]}\left(\mathbb{Z}_{p}[H \backslash G], X^{\prime}\right) \sim \bigoplus_{p^{m_{-} \text {copies }}} X_{k, \lambda} .
$$

By the same way, we have the following equation.

$$
\operatorname{Hom}_{\mathbb{Z}_{p}[G]}\left(\mathbb{Z}_{p}\left[H^{\prime} \backslash G\right], X^{\prime}\right) \sim \bigoplus_{p^{m}-\text { copies }} X_{k^{\prime}, \lambda} .
$$

By Theorem 4,

$$
X^{\prime} \simeq \mathbb{Z}_{p}^{p^{m} \lambda} \oplus \text { finite } p \text {-group. }
$$

Denote by $\psi$ the map from

$$
H o m_{\mathbb{Z}_{p}[G]}\left(\mathbb{Z}_{p}\left[H^{\prime} \backslash G\right], X^{\prime}\right)
$$

to

$$
\operatorname{Hom}_{\mathbb{Z}_{p}[G]}\left(\mathbb{Z}_{p}[H \backslash G], X^{\prime}\right) .
$$

Since $\operatorname{Hom}_{\mathbb{Z}_{p}[G]}\left(A, X^{\prime}\right) \subseteq H o m_{\mathbb{Z}_{p}}\left(A, X^{\prime}\right)$ and the right-hand side is finite, the kernel of the map $\psi$ is finite. The cokernel of the map $\psi$, which is a finitely generated $\mathbb{Z}_{p}$-module, is contained in $\operatorname{Ext}_{\mathbb{Z}_{p}[G]}^{1}\left(A, X^{\prime}\right)$. By definition, $\operatorname{Ext}_{\mathbb{Z}_{p}[G]}^{1}\left(A, X^{\prime}\right)$ is killed by $\# A$. Hence, the cokernel is finite. Therefore we proved that $\bigoplus_{p^{m}-\text { copies }} X_{k^{\prime}, \lambda}$ is pseudo-isomorphic to $\bigoplus_{p^{m} \text {-copies }} X_{k, \lambda}$. This implies, by the structure theorem of $\Lambda$-modules, $X_{k, \lambda}$ is pseudo-isomorphic to $X_{k^{\prime}, \lambda}$. Hence we proved the theorem for $t=m-e$.

Remark. If $p$ does not divide $[L: k]=\left[L: k^{\prime}\right]$, then $X_{k}$ is isomorphic to $X_{k^{\prime}}$. In fact, $p$ does not divide $|H|=\left|H^{\prime}\right|$ in the case, so $\alpha$ in Proposition 4 is zero, and $\mathbb{Z}_{p}[H \backslash G] \simeq \mathbb{Z}_{p}\left[H^{\prime} \backslash G\right]$, so that $A$ is zero in the proof of Theorem 9 . Therefore pseudo-isomorphisms can be replaced by isomorphisms in the above theorems and we can work with $X_{k}$ instead of $X_{k, \lambda}$. Moreover $t=0$ in the case; in other words, $X_{k} \simeq X_{k^{\prime}}$ as $\Lambda_{k}=\Lambda_{k^{\prime}}$-modules.

Remark. The ring $\mathbb{Z}_{p}\left[\operatorname{Gal}\left(L_{\infty} / L\right)\right]=\Lambda_{L}$ can be viewed as a subring $\Lambda_{k, t}$ of

$$
\mathbb{Z}_{p}\left[\operatorname{Gal}\left(k_{\infty} / k\right)\right] \simeq \mathbb{Z}_{p}\left[\operatorname{Gal}\left(k_{\infty}^{\prime} / k^{\prime}\right)\right]=\Lambda_{k}
$$

for some integer $t \geq 0$. The Iwasawa modules $X_{k}$ and $X_{k^{\prime}}$ are actually $\Lambda_{k}$-modules. We showed that $X_{k, \lambda} \sim X_{k^{\prime}, \lambda}$ as a $\Lambda_{L}$-module, not as a $\Lambda_{k}$-module. In general, two 
$\Lambda$-modules which are pseudo-isomorphic as $\Lambda_{t}$-modules are not necessarily pseudoisomorphic as $\Lambda$-modules. Here is an example; let

$$
X=\bigoplus_{p^{t} \text {-copies }} \Lambda / T
$$

and

$$
Y=\Lambda /\left((1+T)^{p^{t}}-1\right) .
$$

They are pseudo-isomorphic to

as

$$
\bigoplus_{p^{t} \text {-copies }} \Lambda_{t} / Z
$$

$$
\Lambda_{t}=\mathbb{Z}_{p}[[Z]]
$$

modules, where $Z=(1+T)^{p^{t}}-1$. However, there is a relation between the characteristic polynomials of two $\Lambda=\mathbb{Z}_{p}[[T]]$-modules which are pseudo-isomorphic as $\Lambda_{t}=\mathbb{Z}_{p}\left[\left[(1+T)^{p^{t}}-1\right]\right]$-modules. By the Weierstrass Preparation Theorem, every power series $f(T) \in \Lambda$ can be expressed by the following way:

$$
f(T)=p^{m} h(T) U(T),
$$

where $h(T)$ is a distinguished polynomial and $U(T)$ is a unit in $\Lambda$. Let $X$ be a finitely generated $\Lambda$-module. When $X$ is considered as a $\Lambda_{t}$-module, we denote it by $X_{t}$, and its characteristic polynomial by $\operatorname{char}_{Z}\left(X_{t}\right)$.

Proposition 6. Let $X$ and $Y$ be finitely generated $\Lambda$-modules and let $\operatorname{char}(X)=$ $p^{\mu_{X}} f_{X}(T)$ and char $(Y)=p^{\mu_{Y}} f_{Y}(T)$. Assume that they are pseudo-isomorphic as $\Lambda_{t}$ modules. Then we have

$$
\mu_{X}=\mu_{Y} \text { and } \prod_{\zeta} f_{X}(\zeta(1+T)-1)=\prod_{\zeta} f_{Y}(\zeta(1+T)-1),
$$

where the product runs through all $p^{t}$-th roots of unity.

Proof. The $\Lambda$-module $\Lambda / p^{m}$ is $\left(\Lambda_{t} / p^{m}\right)^{p^{t}}$ as a $\Lambda_{t}$-module. This proves $\mu_{X}=\mu_{Y}$. Hence, by the structure theorem of $\Lambda$-modules, it is sufficient to prove the theorem in the cyclic case: $X=\Lambda / f^{n}(T)$, where $f(T)$ is irreducible. Let $Z=(1+T)^{p^{t}}-1$. As a $\Lambda_{t}$-module, $X_{t}$ is pseudo-isomorphic to a module of the form $\bigoplus_{i=1}^{s} \Lambda_{t} / f_{i}(Z)$. Consider $\prod_{\zeta} f(\zeta(1+T)-1)$. Then this function is in $\mathbb{Z}_{p}[Z]$. In fact, let $f(T)=$ $\prod_{i=0}^{n}\left(T-\alpha_{i}\right)$; then

$$
\prod_{\zeta} f(\zeta(1+T)-1)=\prod_{i=0}^{n}\left(Z-w_{i}\right)
$$

where $w_{i}=\left(1+\alpha_{i}\right)^{p^{t}}-1$. Then, we know that the $w_{i}$ 's are conjugate to each other. Write $g(Z)=\prod_{\zeta} f(\zeta(1+T)-1)$. Note that $\operatorname{deg}_{T}(f)=\operatorname{deg}_{Z}(g)$ and $f^{n}(T)$ divides $g^{n}(Z)$. Since $f(T)$ is irreducible, $g(Z)$ is a power of an irreducible polynomial $k(Z)$, that is, $g(Z)=k^{d}(Z)$. The module $X_{t}$ is killed by $g^{n}(Z)$, so each $f_{i}(Z)$ divides $g^{n}(Z)$. Hence $f_{i}(Z)$ is a power of the polynomial $k(Z)$. Therefore $\operatorname{char}_{Z}\left(X_{t}\right)=$ $f_{1}(Z) \cdots f_{s}(Z)$ is a power of $k(Z)$. Let $\operatorname{char}_{Z}\left(X_{t}\right)=k^{r}(Z)$. The $\mathbb{Z}_{p}$-rank of $X$ is $n[\operatorname{deg}(f)]$. As a $\Lambda_{t}$-module $X_{t}$, it has the same $\mathbb{Z}_{p}$-rank, that is, $r[\operatorname{deg}(k)]$. Hence we have $r[\operatorname{deg}(k)]=n[\operatorname{deg}(f)]=n[\operatorname{deg}(g)]=n d[\operatorname{deg}(k)]$. From this, we have $r=n d$, so that $\operatorname{char}_{Z}\left(X_{t}\right)=k^{r}(Z)=k^{n d}(Z)=g^{n}(Z)=\prod_{\zeta} f^{n}(\zeta(1+T)-1)$. This completes the proof, since $X_{t}$ and $Y_{t}$ are pseudo-isomorphic as $\Lambda_{t}$-modules, so their characteristic polynomials in $Z$ are the same. 
Remark. W. Sinnott pointed out to me

$$
f_{i}(T)=k(Z)^{n} \quad \text { and } \quad s=\operatorname{deg}_{T} f(T) / \operatorname{deg}_{Z} k(Z) .
$$

The $\mu$-invariant is conjectured to be zero for every basic $\mathbb{Z}_{p}$-extension. Assuming the conjecture, we proved the following statement:

Theorem 10. Assume that $\mu$ is zero for every basic $\mathbb{Z}_{p}$-extension. Let $k$ and $k^{\prime}$ be arithmetically equivalent fields, and $p$ be a prime number. Then

$$
X_{k} \sim X_{k^{\prime}}
$$

as $\Lambda_{L}=\Lambda_{k, t}$-modules for some $t$.

\section{IN THE CM FIELD CASE}

A CM field is a totally imaginary quadratic extension of a totally real number field. Let $k$ be CM, $k_{+}$its maximal real subfield. Let $J$ denote complex conjugation. Fix an odd prime $p$. Recall that $X_{L}$ is the Galois group of the maximal unramified abelian $p$-extension over the basic $\mathbb{Z}_{p}$-extension $L_{\infty}$ of a number field $L$, and $\Lambda=$ $\mathbb{Z}_{p}[[T]]$. Define

$$
X_{k}^{-}=(1-J) X_{k}
$$

In this section, we will prove

Theorem 11. Let $k$ be a CM field, and $k^{\prime}$ be a number field arithmetically equivalent to $k$. Then $k^{\prime}$ is a CM field, and

$$
\operatorname{char}\left(X_{k}^{-}\right) \Lambda=\operatorname{char}\left(X_{k^{\prime}}^{-}\right) \Lambda .
$$

Let $\varepsilon$ be an odd quadratic Artin character of $G a l\left(k / k_{+}\right)$. Write

$$
\begin{aligned}
& \Delta=\operatorname{Gal}\left(k\left(\zeta_{p}\right) / k\right), \\
& e_{0}=1 /|\Delta| \sum_{\delta \in \Delta} \delta .
\end{aligned}
$$

Let $\gamma$ be a topological generator for $\operatorname{Gal}\left(k\left(\zeta_{p^{\infty}}\right) / k\left(\zeta_{p}\right)\right)$, and let $u \in \mathbb{Z}_{p}^{\times}$be such that $\zeta^{\gamma}=\zeta^{u}$ for any $p$-power roots of unity. There exists a quotient of power series $G_{\varepsilon}(T) \in \Lambda$ such that

$$
L_{p}(1-s, \varepsilon \theta)=G_{\varepsilon}\left(u^{s}-1\right),
$$

for $s \in \mathbb{Z}_{p}-\{0\}$. Here the $p$-adic $L$-function $L_{p}(s, \varepsilon \theta)$ is characterized by the following interpolation property:

$$
L_{p}(1-n, \varepsilon \theta)=L_{k_{+}}(1-n, \varepsilon) \prod_{\mathfrak{p} \in S}\left(1-\varepsilon(\mathfrak{p}) N \mathfrak{p}^{n-1}\right),
$$

for $n \equiv 1 \bmod p-1$, where $S$ is the set of primes of $k_{+}$above $p$. To make sense of this recall that for a complex character $\varepsilon$ we can write $L_{k_{+}}(1-n, \varepsilon)$ as a sum

$$
L_{k_{+}}(1-n, \varepsilon)=\sum_{\sigma \in \operatorname{Gal}\left(k / k_{+}\right)} \varepsilon(\sigma) \zeta_{k_{+}}(\sigma, 1-n),
$$

where the partial zeta function $\zeta_{k_{+}}(\sigma, 1-n)$ is a rational number by a result of Klingen and Siegel. By a result of Wiles [15], we have the following

\section{Theorem 12.}

$$
\operatorname{char}\left(e_{0} X_{k\left(\zeta_{p}\right)}\right)^{-} \Lambda=G_{\varepsilon}\left(u(1+T)^{-1}-1\right) \Lambda .
$$


Lemma 5. Let $k$ be a $C M$ field, and $k^{\prime}$ be a number field arithmetically equivalent to $k$. Then $k^{\prime}$ is a CM field, and

$$
\zeta_{k_{+}}=\zeta_{k_{+}^{\prime}} .
$$

Proof. Let $L$ be the Galois closure of $k$. Then $L$ is a CM field. Write $H=\operatorname{Gal}(L / k)$ and $H^{\prime}=\operatorname{Gal}\left(L / k^{\prime}\right)$. Since the complex conjugation $J$ is a center of $\operatorname{Gal}(L / \mathbb{Q})$, the fixed field of $H \times\langle J\rangle$ is the maximal real subfield $k_{+}$. We know that $k^{\prime}$ is totally imaginary because $k^{\prime}$ is arithmetically equivalent to $k$. By assumption, $H$ and $H^{\prime}$ are Gassmann equivalent; hence

$$
\left|c^{G} \cap H\right|=\left|c^{G} \cap H^{\prime}\right|,
$$

for any $c \in G$. Note that $c^{G} \cap H \times\langle J\rangle$ is a disjoint union of $c^{G} \cap H$ and $c^{G} \cap H J$ for any $c \in G$. Since the map given by $g c g^{-1} \rightarrow g c J g^{-1}$ is injective, we have

$$
\left|c^{G} \cap H\right|=\left|(c J)^{G} \cap H J\right| .
$$

Therefore

$$
\begin{aligned}
\left|c^{G} \cap H J\right| & =\left|((c J) J)^{G} \cap H J\right|=\left|(c J)^{G} \cap H\right| \\
& =\left|(c J)^{G} \cap H^{\prime}\right|=\left|(c J J)^{G} \cap H^{\prime} J\right|=\left|c^{G} \cap H^{\prime} J\right| .
\end{aligned}
$$

Hence

$$
\begin{aligned}
\left|c^{G} \cap H\langle J\rangle\right| & =\left|c^{G} \cap H\right|+\left|c^{G} \cap H J\right| \\
& =\left|c^{G} \cap H^{\prime}\right|+\left|c^{G} \cap H^{\prime} J\right|=\left|c^{G} \cap H^{\prime}\langle J\rangle\right| .
\end{aligned}
$$

Therefore, $H\langle J\rangle, H^{\prime}\langle J\rangle$ are Gassmann equivalent, which means the number field $k^{\prime}$ has a totally real subfield $k_{+}^{\prime}$ arithmetically equivalent to $k_{+}$. This completes the proof.

Proof of Theorem 11. By Theorem 12 and the discussion above Theorem 12, $\operatorname{char}\left(e_{0} X_{k\left(\zeta_{p}\right)}\right)^{-}$is determined by $L$-function $L_{k_{+}}(s, \varepsilon)$. By Lemma 5 ,

$$
L_{k_{+}}(s, \varepsilon)=\zeta_{k} / \zeta_{k_{+}}=\zeta_{k^{\prime}} / \zeta_{k_{+}^{\prime}}=L_{k_{+}^{\prime}}(s, \varepsilon) .
$$

This completes the proof by the lemma below.

\section{Lemma 6.}

$$
e_{0} X_{k\left(\zeta_{p}\right)} \simeq X_{k}
$$

Proof. Let $L_{\infty, k\left(\zeta_{p}\right)}$ be the maximal unramified abelian $p$-extension of $k\left(\zeta_{p}\right)_{\infty}$. Let $Y_{0}$ be the subfield of $L_{\infty, k\left(\zeta_{p}\right)}$ fixed by the subgroup $e_{0} X_{k\left(\zeta_{p}\right)}$ of $X_{k\left(\zeta_{p}\right)}$. Since $\operatorname{Gal}\left(k\left(\zeta_{p}\right) / k\right)$ acts trivially on $e_{0} X_{k\left(\zeta_{p}\right)}, Y_{0}$ is the maximal abelian extension of the basic $\mathbb{Z}_{p}$-extension $k_{\infty}$ of $k$ contained in $L_{\infty, k\left(\zeta_{p}\right)}$. Hence the compositum $K_{\infty} L_{\infty, k}$ is contained in $Y_{0}$. Suppose it is properly contained in $Y_{0}$. Then we can construct an unramified abelian $p$-extension $L^{\prime}$ over $k_{\infty}$ properly containing $L_{\infty, k}$ since $p \nmid\left|\operatorname{Gal}\left(k\left(\zeta_{p}\right) / k\right)\right|$, which contradicts the maximality of the extension $L_{\infty, k}$. This completes the proof. 


\section{REFERENCES}

1. N.Adachi and K.Komatsu, The Maximal p-extensions and Zeta-Functions of Algebraic Number Fields, Memoirs of the School of Science \& Engineering Waseda Univ. 51 (1987), 25-31. MR 90a: 11135

2. B. Ferrero and L.Washington, The Iwasawa invariant $\mu_{p}$ vanishes for abelian number fields, Ann. of Math. 109 (1979), 377-395. MR 81a:12005

3. F.Gassmann, Bererkungen zu der vorstehenden arbeit von Hurwitz, Math.Z. 25 (1926), 124143.

4. D.Goss and W.Sinnott, Special Values of Artin L-series, Math.Ann. 275 (1986), 529-537. MR 87k: 11127

5. K.Iwasawa, On p-adic L-functions, Ann. of Math. 89 (1969), 198-205. MR 42:4522

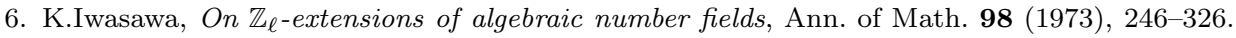
MR 15:509d

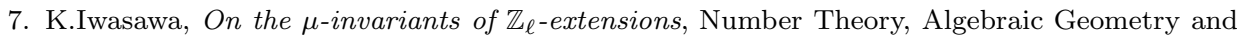
Commutative Algebra (in honor of Y. Akizuki), Kinokuniya, Tokyo, 1973, pp. 1-11. MR 50:9839

8. K.Komatsu, On Zeta-functions and cyclotomic $\mathbb{Z}_{p}$-extensions of algebraic number fields, Tôhoku Math. Journ. 36 (1984), 555-562. MR 86a:11046

9. R.Perlis, On the equation $\zeta_{k}=\zeta_{k^{\prime}}$, J. Number Theory 9 (1977), 342-360. MR 56:5503

10. R.Perlis, On the class numbers of arithmetically equivalent fields, J. Number Theory 10 (1978), 489-509. MR 80c: 12014

11. R.Perlis and N.Colwell, Iwasawa Invariants and Arithmetic Equivalence, unpublished.

12. J.Tate, Endomorphism of abelian varieties over finite fields, Invent. Math. 2 (1966), 134-144. MR 34:5829

13. S.Turner, Adele rings of global field of positive characteristic, Bol. Soc. Brasil. Math. 9 (1978), 89-95. MR 80c: 12017

14. L.Washington, Introduction to Cyclotomic Fields, Springer, Berlin, Heidelberg, New York, 1982. MR 85g: 11001

15. A.Wiles, The Iwasawa conjecture for totally real fields, Ann. of Math. 131 (1990), 493-540. MR 91i: 11163

Department of Mathematics, Ohio State University, Columbus, Ohio 43210

Current address: KIAS, 207-43 Cheongryangri-Dong, Dongdaemun-Gu, Seoul 130-012, Korea

E-mail address: ohj@kias.kaist.ac.kr 